\title{
India CLEN - Annual Meet - 2014
}

\section{The early electroclinical manifestations in children with infantile spasms - a quest for relation to the outcome or etiology - A video EEG study}

Mary Iype, P A Mohammed Kunju, Geetha S, Devi Mohan, Shahanaz Ahamed

Government Medical College, Thiruvanathapuram, Kerala, India

Background: Infantile spasms are an epilepsy syndrome characterized by clustering spasms, and electrographic hypsarrhythmia. Objectives:

- To study the electro clinical characteristics in infantile spasms

- To look for associations of the electro clinical characteristics noted with the outcome and with the etiology

Design: A cross sectional study with prospective follow up

Setting: A tertiary care Pediatric Neurology program

Method: The ictal EEG of 16 consecutive children with infantile spasms, who fulfilled the inclusion criteria, was taken and as part of a long-term study on outcome they were followed up for 3 years Results: 869 spasms were analyzed. Only infantile spasms was seen in 6 (37.5\%), 9 (56.3\%) showed focal seizures and 1 (6.3\%) had multifocal myoclonus in addition to the spasms. Lateralizing phenomena were present in at least some of the spasms in all patients. The commonest ictal EEG correlate was the generalized slow wave transient. The ictal EEG change was consistent from spasm to spasm in the same child. On bivariate analysis, no factor noted on the video EEG analysis had association with seizure freedom, development of autism or the EEG taken at 3years of age and with the etiology.

Conclusion: An infantile spasm is a heterogenous epileptic syndrome and is often associated with seizures other than spasms. On comparison with other studies it was noted that majority of authors could not correlate the etiology or outcome with the ictal EEG. The ictal EEG varied from patient to patient showing that there is no patterned ictal EEG change in infantile spasms.

\section{Carrier state in neonates born to hepatitis b positive mothers-A prospective cohort study}

Geetha S, Dharmarajan S, Santhosh Kumar A, Lalitha Kailas

Department of Pediatrics, Government Medical College, Thiruvanathapuram, Kerala, India

Background: Infection with Hepatitis B virus is a major public health problem. India is the 2nd largest pool of HBV carriers in the world. The transmission of perinatal hepatitis $\mathrm{B}$ infection can be prevented in $70-95 \%$ of neonates by early active immunization and $85-95 \%$ by active \& passive immunoprophylaxis.

Objectives:

- To assess the carrier state in infants born to HBsAg positive mothers at 10th month of age as measured by the HBsAg status.

- To compare the efficacy of prophylaxis as measured by the anti HBs titer at 10th month of age

Design:

- A hospital based prospective cohort study Setting - Tertiary care centre

Methods: Neonates born to HBsAg positive mothers were given $0.5 \mathrm{ml}$ of recombinant vaccine (Revac $-B+$ ) within 12 hours of birth. Hepatitis B Ig was given to only those who could afford it. Hepabig100 i.u. (0.5ml) was given intramuscularly in to the $\mathrm{L}$ thigh within $24 \mathrm{hrs}$ of birth. At 6 and 14 weeks of age, $0.5 \mathrm{ml}$ of recombinant vaccine was given along with other UIP vaccines to all neonates. At 10 months of age (plus 1week) blood samples were drawn. The sera were separated and stored at -20C until tested, Hepacard "Sand wich" immuno assay kit J. was used for HBsAg status determination and electro chemiluminsecent immuno assay using Elecsys 2010 system was used for the estimation of anti HBs titer.

Anti HBs titer $>100 \mathrm{iu} / \mathrm{L}$ was considered as good responder. If anti HBs titer $<10 \mathrm{iu} / \mathrm{L}$ (non responder) the whole vaccine series was repeated by 0, 1, 6 schedule and post vaccination serology done after two months. If the anti HBs titer 10 to 100 , one booster dose of hepatitis vaccine was given and post vaccination serology done after two months.

Results: Total 125 infants were vaccinated with Hep B vaccine in 12 hours. Immunoglobulin was given to 96 infants (76.8\%). Only $69(55.2 \%)$ completed $3^{\text {rd }}$ visit. Carrier state in infants born to Hep B positive mother at 10 months as HBsAg positivity was 1/ $69(1.44 \%) .43 / 69(62.35)$ had good antibody response. Of this 41 were given vaccine\& immunoglobulin. In those given only vaccine2/ $7(28.55)$ had good antibody response. $(p=0.02)$.Geometric mean of antibody in combined group was better. (164.02 compared with 9.75 in vaccine group $\mathrm{p}=0.01$ )

Conclusion: Combined vaccine and Immunoglobulin had better antibody response. The carrier state was $1.44 \%$. There is an absolute need to follow up the babies and check their protection by antibody titre and HBS Ag status at 10 months.

\section{Nurturing the research-the Thrissur way}

T M Ananda Kesavan

Government Medical College, Thrissur, Kerala, India

The author underlined is the Presenting author. 
Aim of the IRC:

- To improve research works among Faculty members, UG and PG students.

- IRC also played a vital role in conducting research oriented CMEs and workshops for the faculty and Students

- IRC helped all the Departments in the campus to conduct the annual CME. It is strictly under the monitoring the IRC.IRC prepared special guidelines regarding how to conduct a CME in the campus(first of its kind)

Results: During the period of 2011 to 2014, a total of 91 studies are funded by SBMR. More than two thirds of the study are completed (all studies of 2014 are ongoing).

7 studies published in National/International journals and a dozen of them are waiting for the review by different Editorial board .Many papers are presented in various state/national/ International conferences.

We observed that the submission of PG thesis to Kerala University of Health and Sciences (KUHS) is in time. The standard of PG dissertation work is improved during the last 2 years and the rejection rate from KUHS is nil.

CME programmes was conducted for PG students (how to write a PG dissertaion) every year and also for faculty members (how to guide a PG dissertation).In addition we conducted CME on statistics, and a CME for exclusively for UG students

IRC collected all the research activities in the campus and published the "Report of the activities"

4 UG students received IMC grant to conduct studies. More than 10 UG students are involved in various research programmes.

5.Planning to have Separate Web site ,Attract more faculty and UG students to the stream line of research, publish/present $60 \%$ of dissertation work of PGs and Publish/present at least one study by PG student in addition to their regular dissertation

\section{Meta-analysis of the efficacy of adjuvant probiotic compared with triple therapy in helicobacter pylori eradication.}

$\underline{\text { Athirarani M R }^{\mathrm{a}}}$, Vrinda Hymavathy ${ }^{\mathrm{b}}$, Sara Varghese ${ }^{\mathrm{c}}$, Regi Jose ${ }^{\mathrm{d}}$

assistant Professor, Government College of Nursing, Thiruvanathapuram, Kerala, India; ${ }^{b}$ RN, NP. Houston Northwest Medical Center, TX, US; 'Government Medical College, Thiruvanathapuram, Kerala, India; ${ }^{\mathrm{d} D e p a r t m e n t}$ of Community Medicine, Sree Gokulam Medical College, Thiruvanathapuram, Kerala, India

Background: Helicobacter Pylori, a gram-negative bacterium found in the stomachs of two-thirds of the world's population. It is responsible for a wide variety of diseases including gastric cancer. It is treated with triple antibiotics and proton pump inhibitors. Despite the treatment, fewer people are being cured than in the past. Studies in the literature have reported that the addition of Probiotics can promote the eradication of $\mathrm{H}$. Pylori.

Objective: To assess the difference in H.pylori eradication rates among patients with H.pylori infection treated with Triple therapy with adjuvant probiotics versus Triple therapy alone in four weeks after the completion of treatment by urea breath test.

Design: Meta analysis

Methods: Electronic databases searched included: Pub Med, CINAHL and Cochrane Database. The review spanned a 14-year period from 2000 to 2014. Key words included in the database search were probiotics, triple therapy, triple therapy and $\mathrm{H}$. pylori eradication. According to the search strategy 135studies matching the key words were identified 131 studies were excluded by title and abstract review. The studies excluded after the matching of objective and methodology were 13 and the studies used for evidentiary meta analysis was 4 .

Results: Analysis was done in REV MAN software from Cochrane. The analysis showed that the overall effect $\mathrm{Z}=6.12(\mathrm{P}<0.00001)$ and pooled $\mathrm{RR}=1.46(1.29,1.64)$

Conclusion: The study showed that the group receiving probiotics along with triple therapy has 1.5 times more chance of H.pylori eradication than the group receiving triple therapy alone.

\section{Prevalence of helminthic infestation among school children}

Premaletha T

Government College of Nursing, Thiruvanathapuram, Kerala, India

Background: Intestinal helminthic infestations are the most common infestations among school age children. The high prevalence of themis closely related to poverty and poor environmental hygiene. They lead to nutritional deficiency and impaired physical development, cognitive function and learning ability. They are the result of inter-related social, economic, cultural, historical and political factors.

Objectives:

- Assess the Prevalence of Helminthic Infestation among School Children

- Find out the association between prevalence of helminthic infestation and the socio demographic and clinical variables.

Methodology:

Approach: Descriptive

Setting: Government upper primary schools Neyyattinkara Thaluk

Population: Students studying in 5th, 6th and 7th standard of upper primary schools of Neyyattinkara Taluk

Sample size: 353

Tools: Structured interview schedule, microscope

Results: Prevalence of helminthic infestation in school children was $44.2 \%$ (95\% confidence limits $38.9-49.5)$, higher in girls (47.5\%) and in coastal area $69.2 \%$ and rural area $37.1 \%$. The most common helminth present in the stool was round worm (68\%).A statistically significant association was found between prevalence of helminthic infestation and place of residence, type of floor of the house, waste disposal, place of defecation, habit of regular deworming and clinical variables such as abdominal pain, vomiting sensation, anorexia and dirt under nail.

Limitation: Ova may not be visible in the single saline smear examination.

Conclusion: The findings of the study indicates that it is time to take collective action to deal with this silent calamity among our children to ensure that they grow up healthy both physically and mentally.

\section{Physical problems and associated factors among stroke survivors}

Asha S Kumar, Joy Philip, Thomas Iype, Rema Devi S, S Muraleedharan Nair 
Government College of Nursing, Medical College, Thiruvananthapuram, Kerala, India

Background: Stroke is a condition with unique epidemiological profile, consisting of high incidence and mortality rates, with a large proportion of survivors experiencing a significant amount of residual disability.

Objectives:

- Estimate the proportion of the physical problems of patients with stroke

- Determine the factors associated with physical problems of stroke survivors

Design: Cross sectional design (hospital based) with case control analysis.

Study setting: Department of Neurology, Medical College, Trivandrum.

Sample: 310 patients who had completed two months since the onset of stroke aged less than 65 years selected by consecutive sampling.

Tool: Stroke Impact Assessment Questionnaire (SIAQ) .

Findings: The proportion of Physical problems of stroke was 66.5 \% with $95 \%$ CI (61.05 to 71.55). Motor problems was $66.1 \%$ (95\% CI 60.83 to 71.36 ), Cognitive problems $65.2 \%$ (95\% CI 59.89 to 70.50 ), Speech problems $66.5 \%$ (95 \% CI 61.24 to 71.75 ) and Sensory problems $65.8 \%$ (95\% CI 60.51 to 71.08 ). Multivariate analysis of significant variables has shown that stroke patients with duration less than one year had 1.7 times and BPL status had 2 times more chance to have physical problems of stroke.

Conclusion: The study highlights the magnitude of stroke mortality and need for detection of these problems early to institute rehabilitation to help them achieve a better quality of life.

\section{CSF CRP estimation in the diagnosis of pyogenic meningitis}

Saji kumar, Rajamohanan

SAT hospital, Thiruvananthapuram, Kerala, India

Background: Pyogenic meningitis continues to be the cause of mortality and Neuro - developmental sequale among children. Early diagnosis is important for prevention of fatality as well as sequale.

Objectives: To study the test properties of CSF CRP among clinically diagnosed \& CSF cytology suggestive meningitis in children against CULTURE or latex positivity considered as Gold Standard and to find the clinical and other lab correlates of CSF CRP Positivity among children with pyogenic meningitis.

Setting: SAT hospital Thiruvananthapuram .

Study subjects: Children who were clinically diagnosed as meningitis \& had cells in CSF were taken for the study.

Sample size: 60 . Calculated based on the proportion of positives $90 \%, 10 \%$ as levels of precision and $\alpha$ level 0.05

Study design: Qualitative assessment of CRP was done by slide latex agglutination method along with biochemistry. CSF Culture or Latex was taken as Gold Standard and the test properties were studied by Descriptive study - Diagnostic Test Evaluation.

Results: CSF CRP had Sensitivity of $95 \%$ and Negative predictive value of $97 \%$,when combined with abnormal biochemistry had positive predictive value $90 \%$ with $95 \%$ specificity.Shock $(p-0.04)$ $\&$ altered sensorium $(\mathrm{p}-0.05)$ had significant correlation with poor outcome in pyogenic meningitis \& CSF CRP positivity. Neutrophilic total count (predominant neutrophils in differential count more than $70 \%$ ) had a significant correlation between CSF CRP positivity. $(p=0.002)$. There was a significant correlation between serum CRP and CSF CRP positivity $(p=0.003)$. CSF CRP had significant correlation with adverse outcome (death and sequale) in pyogenic meningitis with $p$ value 0.049

Conclusion: CSF CRP is effective in ruling out pyogenic meningitis and a cheaper alternative diagnostic test compared to latex and useful in low culture yield settings.

\section{Health related quality of life in patients with type 2 diabetes mellitus}

Sindhu L

Assistant Professor, Government College of Nursing, Thiruvananthapuram, Kerala, India

Introduction: Diabetes mellitus has reached epidemic proportions. According to the World Health Organization (WHO) there is 'an apparent epidemic of diabetes, which is strongly related to lifestyle and economic change.' India has the largest number of diabetics in the world. Diabetes mellitus is a chronic disorder that affects the quality of Life (QOL) of patients.

Objective: Assess the health related Quality of Life of patients with type 2 diabetes mellitus.

Methodology: This was a cross sectional study on 140 type 2 diabetes mellitus patients, attended the diabetic clinic of a Tertiary care centre in Kerala, South India. Patients with type 2 diabetes mellitus between the age group of 25-75 were included in the study .The QoL of patients were assessed using SF 36V2 questionnaire along with the interview schedule for socio demographic and clinical data. The data were analyzed using appropriate descriptive and inferential statistics using SPSS.

Result: The mean age of subjects was $56 \pm 11.6$ years and the mean duration of diabetes mellitus was $10.9 \pm 8.3$ years. $47.9 \%$ of patients were overweight and $20.7 \%$ were obese. The patients with type 2 diabetes had significantly lower scores in all domains of QoL. Males had higher QoL scores than females and the difference was found to be statistically significant $(p=0.006)$.

Conclusion: This study finding indicates that QoL of patients with type 2 diabetes mellitus is relatively poor. Diabetes mellitus significantly affects the QoL especially in females. Therefore much attention must be paid to identify and implement appropriate polices for achieving better management of diabetes mellitus and ultimately improving the QoL of patients with type 2 diabetes mellitus.

\section{Comparison of accuracy of fine needle aspiration cytology and frozen section in the diagnosis of benign and malignant lesions of the breast}

Supriya Menon, Sarath Kumar, Prema N S, Santha Sadasivan

Government Medical College, Thiruvananthapuram, Kerala, India

Background: Frozen section is a vital technique used in breast diagnostics, the most important indications being to confirm the diagnosis of carcinoma and to provide an assessment of resection margins in malignancies. In breast pathology, it allows the surgeon to take an immediate therapeutic decision, sparing a second surgery. 
Objective: To study the accuracy of diagnosis obtained from frozen sections in benign and malignant breast lesions, taking histopathology as gold standard.

Design: Descriptive study with diagnostic test evaluation

Study setting: Department of Pathology, Government Medical College, Thiruvananthapuram, Kerala, India

Methods: One hundred fresh breast specimens after surgery were grossed, frozen sections taken and stained with rapid haematoxylin and eosin. Routine paraffin sections were made subsequently. Diagnosis from frozen section was compared with that of paraffin sections. The accuracy, sensitivity and specificity of frozen section were calculated.

Results: FNAC was done in $89 \%$ of the cases and a positive predictive value of $100 \%$ was obtained. FNAC and histopathology were concordant in $97.8 \%$ of cases. Cohen's kappa of agreement was 0.96 with a $p$ value $<0.0001$ indicating almost perfect agreement. Accuracy of frozen section was $99 \%$, sensitivity and specificity being $98 \%$ and $100 \%$ respectively. Cohen's kappa of agreement was 0.98 with a $p$ value $<0.0001$ indicating almost perfect agreement.

Conclusion: The accuracy of frozen section in diagnosis of malignancy in breast lesions was $99 \%$ and showed excellent concordance with histopathological diagnosis.

\section{Knowledge and attitude of females on male sterilization}

\section{Mini G}

Associate Professor, College of Nursing Ananthapuri Hospitals and Research Institute, Thiruvananthapuram, Kerala, India

Background: In Kerala $49 \%$ of the married females are sterilized and male sterilization is only $3 \%$. In the past, family-planning programs have focused attention primarily on women. WHO expert committee has defined five methods to evaluate the success of family planning methods. One of them is the evaluation of knowledge, attitude, motivation and behaviours among people. Objectives:

- Asses the knowledge and attitude regarding male sterilization among females

- Find out the correlation between knowledge and attitude regarding male sterilization

Methods: The study was descriptive type conducted at selected ward of Trivandrum Corporation selected randomly. Data collected from 100 females selected consecutively. A structured interview schedule was used to assess knowledge and rating scale was used to assess attitude. Data obtained were analyzed by appropriate inferential and descriptive statistics.
Results: $21 \%$ of females had good knowledge, $31 \%$ had poor knowledge. $54 \%$ of females were having good attitude towards male sterilization. $49 \%$ were not agreeing with male sterilization and the major reason were decreased work efficiency of males (38.7\%), fear of complication, (32.6\%) and reduced sexual vigour (4.0\%). There was perfect positive correlation between knowledge and attitude ( $r=1$, P value 0.001$)$ regarding male sterilization.

Conclusion: Family planning service providers and program planners need to be aware of not only the males' knowledge and perceptions pertaining to family planning, but also the attitude of females to enhance the awareness about vasectomy.

\section{Prevalence of ocular morbidity among school children of Perumkadavila block, Thiruvananthapurum district}

Vasantha Mallika M C, SivaSree Ranga M K

Department of Community Medicine, Medical College Karakonam, Kerala, India

Background: In spite of National Programme for Control of Blindness to provide comprehensive health care, ocular morbidity continue to be problem of much Public Health importance. Onethird of India's blind lose their sight before the age of twenty. Early detection and treatment of ocular morbidity among children is important. In Kerala only hospital based data are available. Field based data are scanty.

Objectives: 1.To study the prevalence of ocular morbidity among school children of age group 9-15 years 2 . To find the socio demographic factors associated with ocular morbidity.

Methodology: Cross sectional study among a sample of 1000 students of 9-15 years from schools of Perumkadavila Block for three months from November 2013 . Children are subjected to anthropometric measurements and detailed ocular examination. Collected socio demographic details using pretested proforma. Proportion with confidence interval was estimated to find prevalence. Results are tested by Chi-square test

Results: $36 \%$ of the study group is having one or more preventable or curable form of ocular morbidity of which $24 \%$ with refractive errors. The presence of ocular morbidity showed significant association with demographic variables.

Conclusion: Screening programmes for detecting preventable and treatable causes of defective vision early in life with early interventions will help in preventing progression. The knowledge on distribution and demographic association would enable in planning of eye care services. 